\title{
Notes
}

\section{STRUCTURES OF MINOR COMPONENTS OF THE VALIDAMYCIN COMPLEX}

\author{
Yukihiko Kameda, Naoki Asano, \\ KatsuHiko Matsui, Satoshi Hori ${ }^{\dagger}$ \\ and Hiroshi FuKase ${ }^{\dagger}$
}

School of Pharmacy, Hokuriku University, Kanazawa 920-11, Japan

${ }^{\dagger}$ Central Research Division, Takeda Chemical Industries, Ltd., Osaka 532, Japan

(Received for publication April 9, 1988)

Validamycin A (VM-A), isolated from the culture of Streptomyces hygroscopicus subsp. limoneus, is widely used for the treatment of sheath blight of rice plants and soil-borne plant diseases caused by Bacidiomycetes. The culture broth contains many minor components of the validamycin group in addition to VM-A, the main component.

Since we published the partial structures of the minor components, such as validamycins $\mathbf{B}$ (VM-B), C (VM-C), D (VM-D), E (VM-E) and $\mathrm{F}$ (VM-F), ${ }^{1 \sim 3)}$ structural analysis techniques for complicated oligosaccharides using NMR spectroscopy have developed quite remarkably. The structure of validamycin G (VM-G), ${ }^{4)}$ the most recently discovered congener was established by making use of these techniques. We have now used NMR to reinvestigated the position of the glycosidic linkage of the minor components which had remained obscure.

The results were first disclosed in a patent application, ${ }^{5)}$ though without experimental data. Here we discussed the structures of the minor components with experimental results.

To elucidate the unidentified positions of the glucosidic linkage by ${ }^{1} \mathrm{H}$ NMR spectroscopy, $\mathrm{VM}-\mathrm{B} \sim \mathrm{F}$ were acetylated with acetic anhydride and pyridine to give the corresponding peracetyl derivatives. The chemical shifts of $1-\mathrm{H}$ and $1^{\prime}-\mathrm{H}$ of the peracetates, recognized in the region of $3.2 \sim 3.6 \mathrm{ppm}$, indicate that the imino group is not acetylated. Therefore, these peracetates must be dodeca- $O$-acetyl validamycin B (Ac-

Fig. 1. Structure of the validamycin complex.

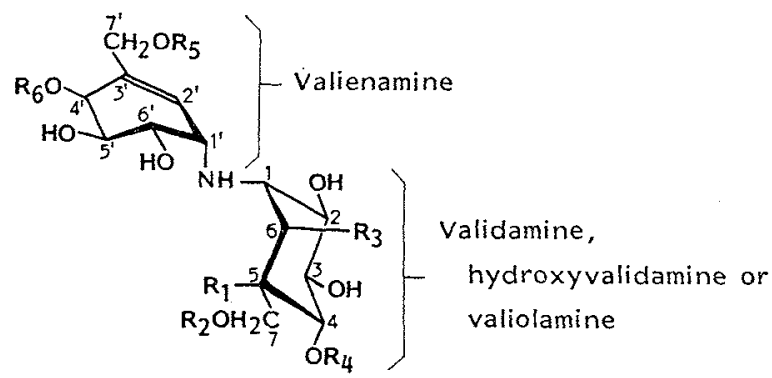

\begin{tabular}{|c|c|c|c|c|c|c|}
\hline Validoxylamine A & $\mathrm{R}_{1}=\mathrm{H}$ & $\mathrm{R}_{2}=\mathrm{H}$ & $\mathrm{R}_{3}=\mathrm{H}$ & $\mathrm{R}_{4}=\mathrm{H}$ & $\mathrm{R}_{5}=\mathrm{H}$ & $\mathrm{R}_{8}=\mathrm{H}$ \\
\hline Validoxylamine B & $\mathrm{R}_{1}=\mathrm{H}$ & $\mathrm{R}_{2}=\mathrm{H}$ & $\mathrm{R}_{3}=\mathrm{OH}$ & $\mathbf{R}_{4}=\mathbf{H}$ & $\mathrm{R}_{5}=\mathrm{H}$ & $\mathrm{R}_{6}=\mathrm{H}$ \\
\hline Validoxylamine G & $\mathrm{R}_{1}=\mathrm{OH}$ & $\mathrm{R}_{2}=\mathrm{H}$ & $\mathrm{R}_{3}=\mathrm{H}$ & $\mathbf{R}_{4}=\mathbf{H}$ & $\mathrm{R}_{5}=\mathrm{H}$ & $\mathbf{R}_{6}=\mathrm{H}$ \\
\hline Validamycin A & $\mathrm{R}_{1}=\mathrm{H}$ & $\mathbf{R}_{2}=\mathbf{H}$ & $\mathrm{R}_{3}=\mathrm{H}$ & $\mathbf{R}_{4}=\beta-\mathrm{D}-\mathrm{Glc}$ & $\mathrm{R}_{5}=\mathrm{H}$ & $\mathbf{R}_{6}=\mathrm{H}$ \\
\hline Validamycin B & $\mathrm{R}_{1}=\mathrm{H}$ & $\mathrm{R}_{2}=\mathrm{H}$ & $\mathrm{R}_{3}=\mathrm{OH}$ & $\mathrm{R}_{4}=\beta$-D-Glc & $\mathrm{R}_{5}=\mathrm{H}$ & $\mathrm{R}_{8}=\mathrm{H}$ \\
\hline Validamycin C & $\mathrm{R}_{1}=\mathrm{H}$ & $\mathrm{R}_{2}=\mathrm{H}$ & $\mathbf{R}_{3}=\mathbf{H}$ & $\mathrm{R}_{4}=\beta$-D-Glc & $\mathrm{R}_{5}=\alpha-\mathrm{D}-\mathrm{Glc}$ & $\mathbf{R}_{\varepsilon}=\mathrm{H}$ \\
\hline Validamycin D & $\mathrm{R}_{1}=\mathrm{H}$ & $\mathrm{R}_{2}=\alpha-\mathrm{D}-\mathrm{Glc}$ & $\mathrm{R}_{3}=\mathrm{H}$ & $\mathbf{R}_{4}=\mathbf{H}$ & $\mathbf{R}_{5}=\mathrm{H}$ & $\mathbf{R}_{6}=\mathrm{H}$ \\
\hline Validamycin $\mathrm{E}$ & $\mathrm{R}_{1}=\mathrm{H}$ & $\mathrm{R}_{2}=\mathrm{H}$ & $\mathrm{R}_{3}=\mathrm{H}$ & $\begin{array}{c}\mathrm{R}_{4}=\alpha-\mathrm{D}-\mathrm{Glc}(1-4)- \\
\beta-\mathrm{D}-\mathrm{Glc}\end{array}$ & $\mathrm{R}_{5}=\mathrm{H}$ & $\mathrm{R}_{6}=\mathrm{H}$ \\
\hline alic & $\mathrm{R}_{1}=\mathrm{H}$ & $\mathrm{R}_{2}=\mathrm{H}$ & $\mathrm{R}_{3}=\mathrm{H}$ & $\mathrm{R}_{4}=\beta-\mathrm{D}-\mathrm{Glc}$ & $\mathrm{R}_{5}=\mathrm{H}$ & -0.6 \\
\hline Validamycin $G$ & $\mathrm{R}_{1}=\mathrm{OH}$ & $\mathrm{R}_{2}=\mathrm{H}$ & $\mathbf{R}_{3}=\mathbf{H}$ & $\mathbf{R}_{4}=\beta-\mathrm{D}-\mathrm{Glc}$ & $\mathrm{R}_{5}=\mathrm{H}$ & $\mathbf{R}_{6}=\mathbf{H}$ \\
\hline
\end{tabular}

Glc: Glucopyranosyl. 
Table $1 .{ }^{13} \mathrm{C}$ NMR spectral data (ppm) of the validamycin components.

\begin{tabular}{|c|c|c|c|c|c|c|c|c|}
\hline Carbon & VA-A & VA-B & VM-D & VM-B & VM-A & VM-C & VM-E & VM-F \\
\hline $\mathrm{C}-1$ & $56.6 \mathrm{~d}$ & $63.1 \mathrm{~d}$ & $56.7 \mathrm{~d}$ & $62.8 \mathrm{~d}$ & $56.3 \mathrm{~d}$ & $56.5 \mathrm{~d}$ & $56.8 \mathrm{~d}$ & $56.4 \mathrm{~d}$ \\
\hline C-2 & $76.4 \mathrm{~d}$ & $73.0^{\mathrm{a}} \mathrm{d}$ & $76.4 \mathrm{~d}$ & $72.3 \mathrm{~d}$ & $75.8 \mathrm{~d}$ & $75.8 \mathrm{~d}$ & $76.1^{\mathrm{a}} \mathrm{d}$ & $75.9^{a} \mathrm{~d}$ \\
\hline $\mathrm{C}-3$ & $77.4 \mathrm{~d}$ & $77.2 \mathrm{~d}$ & $77.1 \mathrm{~d}$ & $75.6 \mathrm{~d}$ & $75.3 \mathrm{~d}$ & $75.3 \mathrm{~d}$ & $75.3 \mathrm{~d}$ & $75.4 \mathrm{~d}$ \\
\hline C- 4 & $76.1 \mathrm{~d}$ & $72.4^{\mathrm{a}} \mathrm{d}$ & $76.2 \mathrm{~d}$ & $85.0 \mathrm{~d}$ & $87.0 \mathrm{~d}$ & $87.0 \mathrm{~d}$ & $86.6 \mathrm{~d}$ & $87.0 \mathrm{~d}$ \\
\hline$C-5$ & $40.6 \mathrm{~d}$ & $45.1 \mathrm{~d}$ & $38.7 \mathrm{~d}$ & $44.6 \mathrm{~d}$ & $40.0 \mathrm{~d}$ & $40.1 \mathrm{~d}$ & $40.1 \mathrm{~d}$ & $40.1 \mathrm{~d}$ \\
\hline C-6 & $29.5 t$ & $70.6 \mathrm{~d}$ & $30.0 \mathrm{t}$ & $71.1 \mathrm{~d}$ & $29.5 \mathrm{t}$ & $29.5 \mathrm{t}$ & $29.1 \mathrm{t}$ & $29.5 \mathrm{t}$ \\
\hline C-7 & $65.1 \mathrm{t}$ & $62.6 \mathrm{t}$ & $71.1 \mathrm{t}$ & $62.7 \mathrm{t}$ & $64.4 \mathrm{t}$ & $64.5 \mathrm{t}$ & $64.2 t$ & $64.5^{\mathrm{b} t}$ \\
\hline C-1' & $55.0 \mathrm{~d}$ & $56.7 \mathrm{~d}$ & $55.0 \mathrm{~d}$ & $56.8 \mathrm{~d}$ & $55.0 \mathrm{~d}$ & $55.1 \mathrm{~d}$ & $55.6 \mathrm{~d}$ & $54.6 \mathrm{~d}$ \\
\hline$C-2^{\prime}$ & $125.9 \mathrm{~d}$ & $125.7 \mathrm{~d}$ & $125.9 \mathrm{~d}$ & $125.7 \mathrm{~d}$ & $125.9 \mathrm{~d}$ & $129.7 \mathrm{~d}$ & $124.9 \mathrm{~d}$ & $128.3 \mathrm{~d}$ \\
\hline $\mathrm{C}-3^{\prime}$ & $141.9 \mathrm{~s}$ & $142.1 \mathrm{~s}$ & $142.0 \mathrm{~s}$ & $142.1 \mathrm{~s}$ & $141.9 \mathrm{~s}$ & $138.4 \mathrm{~s}$ & $142.8 \mathrm{~s}$ & $139.7 \mathrm{~s}$ \\
\hline$C-4^{\prime}$ & $74.1 \mathrm{~d}$ & $74.2 \mathrm{~d}$ & $74.1 \mathrm{~d}$ & $74.2 \mathrm{~d}$ & $74.1 \mathrm{~d}$ & $73.8^{\mathrm{a}} \mathrm{d}$ & $74.0 \mathrm{~d}$ & $80.0 \mathrm{~d}$ \\
\hline $\mathrm{C}-5^{\prime}$ & $76.0 \mathrm{~d}$ & $76.2 \mathrm{~d}$ & $76.0 \mathrm{~d}$ & $76.2^{\mathrm{a}} \mathrm{d}$ & $76.0 \mathrm{~d}$ & $76.3^{b} \mathrm{~d}$ & $76.0^{2} \mathrm{~d}$ & $75.5 \mathrm{~d}$ \\
\hline$C-6^{\prime}$ & $72.0 \mathrm{~d}$ & $72.3^{a} \mathrm{~d}$ & $72.1 \mathrm{~d}$ & $72.3 \mathrm{~d}$ & $72.1 \mathrm{~d}$ & $72.1 \mathrm{~d}$ & $72.0 \mathrm{~d}$ & $72.2 \mathrm{c}$ \\
\hline $\mathrm{C}-7^{\prime}$ & $64.2 \mathrm{t}$ & $64.2 t$ & $64.3 \mathrm{t}$ & $64.2 \mathrm{t}$ & $64.2 \mathrm{t}$ & $69.0 \mathrm{t}$ & $64.2 t$ & $64.6^{b} \mathrm{t}$ \\
\hline $\mathrm{C}-1^{\prime \prime}$ & & & & $105.8 \mathrm{~d}$ & $105.6 \mathrm{~d}$ & $105.6 \mathrm{~d}$ & $105.4 \mathrm{~d}$ & $105.7 \mathrm{~d}$ \\
\hline $\mathrm{C}-2^{\prime \prime}$ & & & & $76.1^{\mathrm{a}} \mathrm{d}$ & $76.3 \mathrm{~d}$ & $76.1^{\mathrm{b}} \mathrm{d}$ & $75.5 \mathrm{~d}$ & $76.1^{\mathrm{a}} \mathrm{d}$ \\
\hline $\mathrm{C}-3^{\prime \prime}$ & & & & $78.3 \mathrm{~d}$ & $78.3 \mathrm{~d}$ & $78.3 \mathrm{~d}$ & $78.7 \mathrm{~d}$ & $78.4 \mathrm{~d}$ \\
\hline$C-4^{\prime \prime}$ & & & & $72.1 \mathrm{~d}$ & $72.1 \mathrm{~d}$ & $72.1 \mathrm{~d}$ & $79.2 \mathrm{~d}$ & $72.2 \mathrm{~d}$ \\
\hline C-5" & & & & $78.7 \mathrm{~d}$ & $78.6 \mathrm{~d}$ & $78.7 \mathrm{~d}$ & $77.3 \mathrm{~d}$ & $78.7 \mathrm{~d}$ \\
\hline C- $6^{\prime \prime}$ & & & & $63.2 \mathrm{t}$ & $63.2 \mathrm{t}$ & $63.2 \mathrm{t}$ & $63.2 \mathrm{t}$ & $63.3 \mathrm{t}$ \\
\hline$C-1^{\prime \prime \prime}$ & & & $100.7 \mathrm{~d}$ & & & $99.0 \mathrm{~d}$ & $102.3 \mathrm{~d}$ & $100.7 \mathrm{~d}$ \\
\hline $\mathrm{C}-2^{\prime \prime \prime}$ & & & $74.1 \mathrm{~d}$ & & & $73.9^{\circ} \mathrm{d}$ & $75.3 \mathrm{~d}$ & $74.8 \mathrm{c}$ \\
\hline $\mathrm{C}-3^{\prime \prime \prime}$ & & & $75.8 \mathrm{~d}$ & & & $75.8 \mathrm{~d}$ & $75.5 \mathrm{~d}$ & $75.7 \mathrm{~d}$ \\
\hline$C-4^{\prime \prime \prime}$ & & & $72.3 \mathrm{~d}$ & & & $72.3 \mathrm{~d}$ & $71.7 \mathrm{~d}$ & $72.2 \mathrm{~d}$ \\
\hline $\mathrm{C}-5^{\prime \prime \prime}$ & & & $74.5 \mathrm{~d}$ & & & $74.6 \mathrm{~d}$ & $74.3 \mathrm{~d}$ & $74.2 \mathrm{~d}$ \\
\hline C- $6^{\prime \prime \prime}$ & & & $63.2 \mathrm{t}$ & & & $63.2 \mathrm{t}$ & $63.2 \mathrm{t}$ & $63.3 \mathrm{t}$ \\
\hline
\end{tabular}

$a, b$ Assignment within any vertical column may be interchanged.

VA, Validoxylamine: VM, validamycin.

VM-B), tetradeca- $O$-acetyl validamycin $\mathrm{C}$ (AcVM-C), undeca- $O$-acetyl validamycin $\mathrm{D}$ (AcVM-D), tetradeca- $O$-acetyl validamycin $\mathrm{E}$ (AcVM-E) and tetradeca-O-acetyl validamycin $F$ (Ac-VM-F), respectively.

The ${ }^{13} \mathrm{C}$ NMR spectra of the validamycins were recorded with a Jeol JNM-FX 100 spectrometer at $25.2 \mathrm{MHz}$ in $\mathrm{D}_{2} \mathrm{O}$, with sodium 2,2-dimethyl2-silapentane-5-sulfonate as an internal standard; data are summarized in Table 1 . The ${ }^{1} \mathrm{H}$ NMR spectra of the validamycin peracetates were recorded with a Jeol JNM-GX400 spectrometer at $400 \mathrm{MHz}$ in $\mathrm{CDCl}_{3}+\mathrm{D}_{2} \mathrm{O}$, with TMS as an internal standard; data are summarized in Table 2. 2D Correlation spectroscopy (2D COSY) experiments were performed with data matrices of $512 \times 2,048$ data points, using the standard Jeol software.

\section{VMs-C, E and F}

Previous studies $\left.{ }^{1}, 2\right)$ have shown that VMs-C, $E$ and $F$ are pseudo-tetrasaccharides which each gave one mole of validoxylamine A (VA-A) and $2 \mathrm{~mol}$ of D-glucose by complete acid hydrolysis and VM-A by partial acid hydrolysis or selective hydrolysis using microbial $\alpha$-glucosidase. $\mathrm{Hy}$ drogenolysis of VMs-C and $\mathrm{F}$ affords $4-O-\beta-\mathrm{D}-$ glucopyranosyl-validamine and $\alpha$-D-glucopyranosyl-validatol as a result of $\mathrm{C}-\mathrm{N}$ bond cleavage of allyl system. However, the $\alpha$-D-glucopyranosyl-validatols derived from VM-C and VM-F differ from one another in the NMR spectrum and gas-liquid chromatography (GLC) analysis of their TMS-derivatives. VMs-C and $F$, therefore differ only in the position of attachment of D-glucose to the valienamine part of the VA-A unit. Hydrogenolysis of VM$\mathrm{E}$ affords $\alpha$-D-glucopyranosyl-(4- $O-\beta$-D-glucopyranosyl) validamine and validatol; additionally, maltose was detected in a partial acid hydrolysate of VM-E.

${ }^{13} \mathrm{C}$ NMR Studies: Complete signal assignment of VM-A was made by WeN-ZAO et al. ${ }^{8)}$ and is in agreement with our analysis. The 
Table 2. ${ }^{1} \mathrm{H}$ NMR spectral data of the per- $O$-acetates of validamycin complex $(\delta$ in ppm, $J$ in $\mathrm{Hz})$.

\begin{tabular}{|c|c|c|c|c|}
\hline Proton & Validoxylamine A acetate & Validamycin $\mathrm{D}$ acetate & Validoxylamine B acetate & Validamycin B acetate \\
\hline $1-\mathrm{H}$ & $3.370\left(\mathrm{q}^{a}, J=2.4,3.5,3.9\right)$ & $3.395\left(\mathrm{q}^{\mathrm{a}}, J=2.5,3.4,3.9\right)$ & $3.309\left(t^{a}, J=3.9,4.6\right)$ & $3.224\left(\mathrm{t}^{\mathrm{a}}, J=3.5,4.6\right)$ \\
\hline $2-\mathrm{H}$ & $4.939(\mathrm{dd}, J=3.9,10.5)$ & $4.966(\mathrm{dd}, J=3.9,10.5)$ & $5.204(\mathrm{dd}, J=4.6,10.5)$ & $5.128(\mathrm{dd}, J=4.6,10.3)$ \\
\hline 3-H & $5.397(\mathrm{dd}, J=9.5,10.5)$ & $5.411(\mathrm{dd}, J=9.5,10.5)$ & $5.345(\mathrm{dd}, J=9.3,10.5)$ & $5.307(\mathrm{dd}, J=9.0,10.3)$ \\
\hline $4-\mathrm{H}$ & $4.972(\mathrm{dd}, J=9.5,10.5)$ & $5.047(\mathrm{t}, J=9.5)$ & $5.214(\mathrm{dd}, J=9.3,11.2)$ & $3.782(\mathrm{dd}, J=9.0,10.9)$ \\
\hline $5-\mathrm{H}$ & $2.39 \sim 2.50(\mathrm{~m})$ & $2.32 \sim 2.43(\mathrm{~m})$ & $2.70 \sim 2.78\left(\mathrm{~m}, J_{4,5}=11.2^{\mathrm{b}}\right)$ & $2.62 \sim 2.72\left(\mathrm{~m}, J_{4,5}=10.9^{\mathrm{b}}\right)$ \\
\hline $6-\mathrm{H}$ & & & $5.182\left(\mathrm{t}^{\mathrm{a}}, J=3.0,3.9\right)$ & $5.129\left(\mathrm{t}^{\mathrm{a}}, J=3.0,3.5\right)$ \\
\hline $6-\mathrm{H}_{\mathrm{ax}}$ & $1.515(\mathrm{ddd}, J=2.4,12.5,14.7)$ & $1.629(\mathrm{ddd}, J=2.5,12.5,14.7)$ & & \\
\hline $6-\mathrm{H}_{\mathrm{eq}}$ & $1.841(\mathrm{td}, J=3.5,3.5,14.7)$ & $1.792(\mathrm{td}, J=3.4,3.4,14.7)$ & & \\
\hline $7-\mathrm{H}_{\mathrm{a}}$ & $3.884(\mathrm{dd}, J=3.3,11.4)$ & $3.234(\mathrm{dd}, J=3.9,9.8)$ & $3.940(\mathrm{dd}, J=4.4,11.2)$ & $4.159\left(\mathrm{t}^{\mathrm{a}}, J=10,10.7\right)$ \\
\hline $7-\mathrm{H}_{\mathrm{b}}$ & $4.131(\mathrm{dd}, J=4.8,11.4)$ & $3.728(\mathrm{dd}, J=3.7,9.8)$ & $4.211(\mathrm{dd}, J=8.6,11.2)$ & $4.297(\mathrm{dd}, J=4.2,10.7)$ \\
\hline $1^{\prime}-\mathrm{H}$ & $3.534\left(\mathrm{t}^{\mathrm{a}}, J=4.6,4.9\right)$ & $3.582(\mathrm{t}, J=4.6)$ & $3.579\left(\mathrm{t}^{\mathrm{a}}, J=4.6,4.9\right)$ & $3.596\left(\mathrm{t}^{\mathrm{a}}, J=4.6,4.9\right)$ \\
\hline $2^{\prime}-\mathrm{H}$ & $5.991(\mathrm{dd}, J=1.2,4.6)$ & $5.994(\mathrm{dd}, J=1.0,4.6)$ & $5.994(\mathrm{dd}, J=1.0,4.9)$ & $5.997(\mathrm{dd}, J=1.0,4.9)$ \\
\hline $4^{\prime}-\mathrm{H}$ & $5.491($ br d, $J=6.1)$ & $5.489($ br d, $J=6.1)$ & $5.500($ br d, $J=6.3)$ & $5.510(\mathrm{br} \mathrm{d}, J=6.3)$ \\
\hline $5^{\prime}-\mathrm{H}$ & $5.416(\mathrm{dd}, J=6.1,9.5)$ & $5.407(\mathrm{dd}, J=6.1,9.5)$ & $5.416(\mathrm{dd}, J=6.3,9.8)$ & $5.404(\mathrm{dd}, J=6.3,9.8)$ \\
\hline $6^{\prime}-\mathrm{H}$ & $4.985(\mathrm{dd}, J=4.9,9.5)$ & $4.981(\mathrm{dd}, J=4.6,9.5)$ & $5.019(\mathrm{dd}, J=4.6,9.8)$ & $4.989(\mathrm{dd}, J=4.6,9.8)$ \\
\hline $7^{\prime}-\mathrm{H}_{\mathrm{a}}$ & $4.391(\mathrm{~d}, J=13.2)$ & $4.400(\mathrm{~d}, J=13.1)$ & $4.385(\mathrm{~d}, J=13.2)$ & $4.387(\mathrm{~d}, J=13.2)$ \\
\hline $7^{\prime}-\mathrm{H}_{\mathrm{b}}$ & $4.653(\mathrm{dd}, J=1.2,13.2)$ & $4.664(\mathrm{dd}, J=1.0,13.1)$ & $4.653(\mathrm{dd}, J=1.0,13.2)$ & $4.661(\mathrm{dd}, J=1.0,13.2)$ \\
\hline $1^{\prime \prime}-\mathrm{H}$ & & & & $4.503(\mathrm{~d}, J=8.1)$ \\
\hline $2^{\prime \prime}-\mathrm{H}$ & & & & $4.947(\mathrm{dd}, J=8.1,9.3)$ \\
\hline $3 \prime-H$ & & & & $5.152(\mathrm{t}, J=9.3)$ \\
\hline $4^{\prime \prime}-\mathrm{H}$ & & & & $5.098(\mathrm{t}, J=9.3)$ \\
\hline $5^{\prime \prime}-\mathrm{H}$ & , & & & $3.680(\mathrm{ddd}, J=2.2,3.9,9.3)$ \\
\hline $6^{\prime \prime}-\mathrm{H}_{\mathrm{a}}$ & & & & $4.037(\mathrm{dd}, J=2.2,12.5)$ \\
\hline $6^{\prime \prime}-\mathrm{H}_{\mathrm{b}}$ & & & & $4.433(\mathrm{dd}, J=3.9,12.5)$ \\
\hline $1^{\prime \prime \prime}-\mathrm{H}$ & & $5.000(\mathrm{~d}, J=3.8)$ & & \\
\hline $2^{\prime \prime \prime}-\mathrm{H}$ & & $4.864(\mathrm{dd}, J=3.8,10.2)$ & & \\
\hline $3^{\prime \prime \prime}-H$ & & $5.432(\mathrm{dd}, J=9.2,10.2)$ & & \\
\hline $4^{\prime \prime \prime}-\mathrm{H}$ & & $5.074(\mathrm{t}, J=9.2)$ & & \\
\hline $5^{\prime \prime \prime}-\mathrm{H}$ & & $4.021(\mathrm{ddd}, J=2.2,3.7,9.2)$ & & \\
\hline $6^{\prime \prime \prime}-\mathrm{H}_{\mathrm{a}}$ & & $4.008(\mathrm{dd}, J=2.2,12.5)$ & & \\
\hline $6^{\prime \prime \prime}-\mathrm{H}_{\mathrm{b}}$ & & $4.302(\mathrm{dd}, J=3.7,12.5)$ & & \\
\hline Acetyl & $\begin{array}{l}2.001,2.035,2.063,2.065 \\
2.067,2.073,2.078,2.089\end{array}$ & $\begin{array}{l}2.016,2.018,2.046,2.052 \\
2.063,2.070,2.075,2.075 \\
2.080,2.089,2.094\end{array}$ & $\begin{array}{l}2.018,2.040,2.049,2.062 \\
2.068,2.070,2.075,2.123 \\
2.130\end{array}$ & $\begin{array}{l}1.987,2.012,2.032,2.048 \\
2.062,2.067,2.068,2.068 \\
2.075,2.087,2.106,2.117\end{array}$ \\
\hline
\end{tabular}


Table 2. (Continued)

\begin{tabular}{|c|c|c|c|c|}
\hline Proton & Validamycin $\mathrm{A}$ acetate & Validamycin $\mathrm{C}$ acetate & Validamycin $\mathbf{E}$ acetate & Validamycin $F$ acetate \\
\hline $1-\mathrm{H}$ & $3.262\left(\mathrm{q}^{\mathrm{z}}, J=2.0,3.5,3.9\right)$ & $3.273\left(\mathrm{q}^{\mathrm{a}}, J=2.0,3.3,3.8\right)$ & $3.265\left(\mathrm{q}^{a}, J=2.0,3.0,3.5\right)$ & $3.352\left(\mathrm{q}^{\mathrm{a}}, J=2.5,3.0,3.9\right)$ \\
\hline $2-\mathrm{H}$ & $4.870(\mathrm{dd}, J=3.9,10.3)$ & $4.874(\mathrm{dd}, J=3.8,10.1)$ & $4.863(\mathrm{dd}, J=3.5,10.3)$ & $4.909(\mathrm{dd}, J=3.9,10.3)$ \\
\hline 3-H & $5.346(\mathrm{dd}, J=9.0,10.3)$ & $5.345(\mathrm{dd}, J=9.3,10.1)$ & $5.347(\mathrm{dd}, J=9.3,10.3)$ & $5.406(\mathrm{dd}, J=9.3,10.3)$ \\
\hline 4-H & $3.583(\mathrm{dd}, J=9.0,10.2)$ & $3.580(\mathrm{dd}, J=9.3,10.2)$ & $3.591(\mathrm{dd}, J=9.3,10.5)$ & $3.599(\mathrm{dd}, J=9.3,10.7)$ \\
\hline $5-\mathrm{H}$ & $2.26 \sim 2.37(\mathrm{~m})$ & $2.25 \sim 2.34(\mathrm{~m})$ & $2.26 \sim 2.36(\mathrm{~m})$ & $2.33 \sim 2.44(\mathrm{~m})$ \\
\hline $6-H_{a x}$ & $1.374(\mathrm{ddd}, J=2.0,12.2,14.6)$ & $1.397(\mathrm{ddd}, J=2.0,12.0,14.2)$ & $1.375(\mathrm{ddd}, J=2.0,12.0,14.2)$ & $1.400(\mathrm{ddd}, J=2.5,12.0,14.6)$ \\
\hline $6-\mathrm{H}_{\mathrm{eq}}$ & $1.800(\mathrm{td}, J=3.5,3.5,14.6)$ & $1.812(\mathrm{td}, J=3.3,3.3,14.2)$ & $1.794(\mathrm{td}, J=3.0,3.0,14.2)$ & $1.731\left(\mathrm{td}^{\mathrm{a}}, J=3.0,3.5,14.6\right)$ \\
\hline $7-\mathrm{H}_{\mathrm{a}}$ & $4.034(\mathrm{dd}, J=2.2,12.5)$ & $4.129(\mathrm{dd}, J=4.9,11.2)$ & $4.124(\mathrm{dd}, J=4.9,11.2)$ & $4.118(\mathrm{dd}, J=4.0,11.2)$ \\
\hline $7-\mathrm{H}_{\mathrm{b}}$ & $4.394(\mathrm{dd}, J=3.9,12.5)$ & $4.309(\mathrm{dd}, J=2.9,11.2)$ & $4.305(\mathrm{dd}, J=2.5,11.2)$ & $4.333(\mathrm{dd}, J=2.7,11.2)$ \\
\hline $1^{\prime}-\mathrm{H}$ & $3.558\left(\mathrm{t}^{\mathrm{a}}, J=4.5,4.9\right)$ & $3.584\left(\mathrm{t}^{\mathrm{a}}, J=4.2,4.4\right)$ & $3.552\left(\mathrm{t}^{\mathrm{a}}, J=4.5,4.8\right)$ & $3.48 \sim 3.52(\mathrm{~m})$ \\
\hline $2^{\prime}-\mathrm{H}$ & $5.962(\mathrm{dd}, J=1.0,4.5)$ & $5.986(\mathrm{~d}, J=4.2)$ & $5.962(\mathrm{dd}, J==1.0,4.5)$ & $5.869\left(\right.$ br s $\left.^{a}\right)$ \\
\hline $4^{\prime}-\mathrm{H}$ & $5.497($ br d,$J=6.1)$ & $5.332($ br d, $J=5.4)$ & $5.488($ br d, $J=6.1)$ & $3.935\left(\mathrm{br} \mathrm{s}{ }^{a}, J_{4^{\prime}, 5^{\prime}}=2.0^{\mathrm{b}}\right)$ \\
\hline $5^{\prime}-\mathrm{H}$ & $5.405(\mathrm{dd}, J=6.1,9.5)$ & $5.401(\mathrm{dd}, J=5.4,8.8)$ & $5.394(\mathrm{dd}, J=6.1,9.4)$ & $5.140(\mathrm{dd}, J=2.0,4.9)$ \\
\hline $6^{\prime}-\mathrm{H}$ & $4.966(\mathrm{dd}, J=4.9,9.5)$ & $4.965(\mathrm{dd}, J=4.4,8.8)$ & $4.971(\mathrm{dd}, J=4.8,9.4)$ & $4.996($ br t $, J=4.1,4.9)$ \\
\hline $7^{\prime}-\mathrm{H}_{\mathrm{a}}$ & $4.391(\mathrm{~d}, J=13.5)$ & $3.879(\mathrm{~d}, J=12.7)$ & $4.388(\mathrm{brd}, J=13.2)$ & $4.604\left(2 \mathrm{H}\right.$, br s$\left.^{\mathrm{a}}\right)$ \\
\hline $7^{\prime}-\mathrm{H}_{\mathrm{b}}$ & $4.659($ br d, $J=13.5)$ & $4.184($ br d, $J=12.7)$ & $4.654(\mathrm{dd}, J=1.0,13.2)$ & \\
\hline $1^{\prime \prime}-\mathrm{H}$ & $4.520(\mathrm{~d}, J=7.8)$ & $4.512(\mathrm{~d}, J=8.1)$ & $4.572(\mathrm{~d}, J=7.8)$ & $4.525(\mathrm{~d}, J=8.1)$ \\
\hline $2^{\prime \prime}-\mathrm{H}$ & $4.944(\mathrm{dd}, J=7.8,9.0)$ & $4.939(\mathrm{dd}, J=8.1,9.3)$ & $4.777(\mathrm{dd}, J=7.8,8.8)$ & $4.945(\mathrm{dd}, J=8.1,9.1)$ \\
\hline $3^{\prime \prime}-\mathrm{H}$ & $5.153\left(\mathrm{t}^{\mathrm{a}}, J=9.0,9.3\right)$ & $5.152(\mathrm{t}, J=9.3)$ & $5.184(\mathrm{t}, J=-8.8)$ & $5.153\left(\mathrm{t}^{\mathrm{a}}, J=9.1,9.6\right)$ \\
\hline $4^{\prime \prime}-\mathrm{H}$ & $5.084\left(\mathrm{t}^{\mathrm{a}}, J=9.3,9.8\right)$ & $5.078\left(\mathrm{t}^{\mathrm{a}}, J=9.3,9.8\right)$ & $3.998(\mathrm{dd}, J=8.8,9.4)$ & $5.089(\mathrm{t}, J=9.6)$ \\
\hline $5^{\prime \prime}-\mathrm{H}$ & 3.645 (ddd, $J=2.6,4.0,9.8)$ & $3.645(\mathrm{ddd}, J=2.2,4.2,9.8)$ & $3.674(\mathrm{ddd}, J=2.6,3.9,9.4)$ & $3.646(\mathrm{ddd}, J=2.2,4.2,9.6)$ \\
\hline $6^{\prime \prime}-\mathrm{H}_{\mathrm{a}}$ & $4.135(\mathrm{dd}, J=4.0,11.2)$ & $4.034(\mathrm{dd}, J=2.2,12.5)$ & $4.268(\mathrm{dd}, J=3.9,12.3)$ & $4.042(\mathrm{dd}, J=2.2,12.5)$ \\
\hline $6^{\prime \prime}-\mathrm{H}_{\mathrm{b}}$ & $4.306(\mathrm{dd}, J=2.6,11.2)$ & $4.389(\mathrm{dd}, J=4.2,12.5)$ & $4.339(\mathrm{dd}, J=2.6,12.3)$ & $4.401(\mathrm{dd}, J=4.2,12.5)$ \\
\hline $1^{\prime \prime \prime}-\mathrm{H}$ & & $5.058(\mathrm{~d}, J=3.7)$ & $5.374(\mathrm{~d}, J=3.9)$ & $5.290(\mathrm{~d}, J=3.9)$ \\
\hline $2^{\prime \prime \prime}-\mathrm{H}$ & & $4.842(\mathrm{dd}, J=3.7,10.3)$ & $4.854(\mathrm{dd}, J=3.9,10.6)$ & $5.029(\mathrm{dd}, J=3.9,10.5)$ \\
\hline $3^{\prime \prime \prime}-\mathrm{H}$ & & $5.454(\mathrm{dd}, J=9.5,10.3)$ & $5.347(\mathrm{dd}, J=9.8,10.6)$ & $5.370(\mathrm{dd}, J=9.6,10.5)$ \\
\hline $4^{\prime \prime \prime}-\mathrm{H}$ & & $5.053\left(\mathrm{t}^{\mathrm{a}}, J=9.5,10.5\right)$ & $5.054(\mathrm{t}, J=9.8)$ & $5.092(\mathrm{t}, J=9.6)$ \\
\hline $5^{\prime \prime \prime}-\mathrm{H}$ & & $3.967(\mathrm{ddd}, J=2.2,4.4,10.5)$ & $3.924(\mathrm{ddd}, J=2.2,3.5,9.8)$ & $4.10 \sim 4.15(\mathrm{~m})$ \\
\hline $6^{\prime \prime \prime}-\mathrm{H}_{\mathrm{a}}$ & & $4.096(\mathrm{dd}, J=2.4,12.5)$ & $4.025(\mathrm{dd}, J=2.2,12.5)$ & $4.124(\mathrm{brd}, J=12.6)$ \\
\hline $6^{\prime \prime \prime}-\mathrm{H}_{\mathrm{b}}$ & & $4.268(\mathrm{dd}, J=4.2,12.5)$ & $4.223(\mathrm{dd}, J=3.5,12.5)$ & $4.233(\mathrm{dd}, J=4.2,12.6)$ \\
\hline Acetyl & $\begin{array}{l}1.988,2.007,2.011,2.052 \\
2.059,2.060,2.068,2.072 \\
2.072,2.083,2.116\end{array}$ & $\begin{array}{l}1.987,2.001,2.007,2.007 \\
2.029,2.057,2.058,2.062 \\
2.079,2.079,2.088,2.103 \\
2.113,2.117\end{array}$ & $\begin{array}{l}1.990,1.992,2.001,2.024 \\
2.034,2.046,2.050,2.059 \\
2.062,2.067,2.067,2.098 \\
2.135,2.155\end{array}$ & $\begin{array}{l}1.988,1.998,2.009,2.010 \\
2.018,2.050,2.057,2.085 \\
2.085,2.107,2.112,2.123 \\
2.123,2.168\end{array}$ \\
\hline
\end{tabular}

${ }^{a}$ Splitting pattern in appearance. $\mathrm{b}$ Revealed by decoupling experiment. 
signals of the $\alpha$-D-glucopyranose unit and the glucosyloxylated carbon in the VM-A unit of VMs-C and $\mathrm{F}$ were easily assigned by comparing their spectra with those of VM-A (Table 1). Their shift patterns consistent with the known effects of glycosidation $\left.{ }^{7}, 8\right)$ and the structures depicted in Fig. 1.

${ }^{1} \mathrm{H}$ NMR Studies: The coupling networks of Ac-VM-C and Ac-VM-E were unraveled by 2D COSY experiments, except for the overlapping 4-H and $1^{\prime}-\mathrm{H}$ signals which were resolved by irradiation at $5-\mathrm{H}$ and $2^{\prime}-\mathrm{H}$ in a standard spin decoupling experiment. The sequence of AcVM-F was performed entirely by a standard spin decoupling experiment (Table 2). The positions of glucosidic linkage were confirmed by the relative high-field shift of the protons linked to glucosyloxylated carbons compared with the signals of the corresponding protons linked to acetoxylated carbons. Data in Table 2 are consistent with the structures shown in Fig. 1.

\section{VMs-B and D}

${ }^{1} \mathrm{H}$ NMR Studies: The glycosidation upfield chemical shift $(1.43 \mathrm{ppm})$ of the $4-\mathrm{H}$ signal $(\delta$ 3.782) of the validamine unit of Ac-VM-B was recognized in comparison with 4-H ( $\delta$ 5.214) of nona- $O$-acetyl validoxylamine B (Ac-VA-B), which could be assigned by chemical shift and spin decoupling experiments. In the case of Ac-VM-D, a glycosidation upfield shift was observed at a pair of doublet of doublets signals at $\delta 3.234$ and 3.728 which was assigned to $7-\mathrm{H}$ of the validamine unit, compared with the 7-H signals ( $\delta 3.884$ and 4.131$)$ of Ac-VA-A.

${ }^{13} \mathrm{C}$ NMR Studies: The position of glucosidic linkage in VM-D was confirmed to be at C-7 of the validamine unit on the basis of a $6.0 \mathrm{ppm}$ downfield shift of the $\alpha$-carbon (C-7) and a 1.9ppm upfield shift for the $\beta$-carbon (C-5), compared with those of VA-A.

From the above results, we elucidated and confirmed the position of glucosidic linkages of minor components of the validamycin group as shown in Fig. 1.

With regard to structure-activity relationships, it is apparent that the 4-O- $\beta$-D-glucosyl residue of VM-A plays a very important role and causes the remarkable increment of permeability of the antibiotic into the mycelium of the pathogen, Rhizoctonia solani. ${ }^{\text {})}$ The 7-O- and $7^{\prime}-O-\alpha-\mathrm{D}-$ glucosyl residues of VM-C and VM-D cause deleterious effects on the activity, whereas the $4^{\prime}-O-\alpha$-D-glucosyl residue of VM-F and the $4^{\prime \prime}$ $O-\alpha$-D-glucosyl residue of VM-E have little effect.

\section{References}

1) Kameda, Y.; S. Horit \& T. Yamano: Microbial transformation of validamycins. J. Antibiotics 28: 298 306, 1975

2) Horil, S.; Y. Kameda \& K. Kawahara: Studies on validamycins, new antibiotics. VIII. Isolation and characterization of validamycins C, D, E and F. J. Antibiotics 25: 48 53, 1972

3) Horir, S.; T. IwasA \& Y. KaMeda: Studies on validamycins, new antibiotics. V. Degradation studies. J. Antibiotics 24: 57 58, 1971

4) Kameda, Y.; N. Asano, T. Yamaguchi, K. Matsui, S. Horil \& H. Fukase: Validamycin $G$ and validoxylamine $G$, new members of the validamycins. J. Antibiotics 39: $1491 \sim 1494$, 1986

5) KamedA, Y. \& S. HoRII (Hokuriku Univ. \& Takeda): Valiolamine derivatives and production thereof. Jpn. Kokai 48692('87), 1987 and extra application volume $3(2)-36(661)$ pp. 7, 14 in supplemental part, May 13, 1987

6) JIN, WEN-ZAO; K. L. RINEHART, Jr. \& T. TOYOKUNI: Biosynthetic studies on validamycins. I. ${ }^{1} \mathrm{H}$ and ${ }^{13} \mathrm{C}$ NMR assignments of validamycin A. J. Antibiotics 40: 329 339, 1987

7) YaMASAKI, K.; H. KoHDA, T. KobaYashi, R. KaSAI \& O. TANAKA: Structures of stevia diterpene-glucosides: Application of ${ }^{18} \mathrm{C}$ NMR. Tetrahedron Lett. 1976: 1005 1008, 1976

8) Yokose, K.; K. OGAWA, Y. SUzUKI, I. UMEdA \& Y. SumaRa: New $\alpha$-amylase inhibitor, trestatins. II. Structure determination of trestatins A, B and C. J. Antibiotics 36: 1166 1175, 1983

9) Asano, N.; T. Yamaguchi, Y. Kameda \& K. MATSUI: Effect of validamycins on glycohydrolases of Rhizoctonia solani. J. Antibiotics 40: $526 \sim 532,1987$ 